\title{
Hängt der Psychotherapieerfolg doch nicht vom Einkommen ab?
}

\author{
Beobachtungen weisen auf einen geringeren Erfolg psychotherapeutischer Interventionen bei Patienten mit einem \\ niedrigen Einkommen als bei Patienten mit hohem Einkommen hin. Das konnte hier nicht bestätigt werden.
}

\begin{abstract}
A ngststörungen haben eine hohe Prävalenz. Die Hypothese, dass der Erfolg psychotherapeutischer Interventionen bei Patienten mit einem niedrigen Einkommen geringer ist als bei Patienten mit hohem Einkommen, wird durch zahlreiche systematische Beobachtungen gestützt. Der geringere Therapieerfolg wird damit begründet, dass geringverdienende Patienten während der Intervention weiterhin vergleichsweise hohem ökonomischen Stress ausgesetzt seien. In diese Studie wurden 287 Patienten mit geringem und 717 mit hohem Einkommen, die unter einer Panikstörung, einer sozialen Angststörung, einer generalisierten Angst- oder einer posttraumatischen Belastungsstörung litten, eingeschlossen. Die Patienten wurden im Rah-
\end{abstract}

men von CALM (Coordinated Anxiety Learning and Management) behandelt. CALM hält in der Primärversorgung verschiedene Module (im Wesentlichen Beratung, Medikation, KVT) vor, die - je nach Bedarf - isoliert oder in Kombination über einen Zeitraum von zehn bis zwölf Wochen eingesetzt werden.

Es zeigte sich, dass die Patienten mit einem geringen Einkommen vor CALM eine schwerere Angstsymptomatik aufwiesen im Vergleich zu Patienten mit hohem Einkommen. Der Therapieerfolg, gemessen als Ausmaß der Reduktion der Angstsymptomatik im Präpost-Vergleich, war aber - die Haupthypothese dieser Studie widerlegend in beiden Gruppen gleich ausgeprägt. Da die Patienten mit niedrigem Ein- kommen allerdings einen höheren initialen Angstwert aufweisen, waren sie auch nach CALM deutlicher krank als Patienten mit hohem Einkommen.

Kommentar: Diese Studie zeigt uns, dass es darauf ankommt, auch Menschen mit prekärer ökonomischer Situation eine adäquate Therapie zukommen zu lassen, da der Therapieerfolg ebenso groß wie bei Patienten mit höherem Einkommen ist. Allerdings sollten sie auch eine ähnlich intensive Therapie erhalten.

Prof. Dr. med. Hans-Peter Volz

Sullivan $\mathrm{G}$ et al. Does a quality improvement intervention for anxiety result in differential outcomes for lower income patients? Am J Psychiatry 2013; 170 (2): 218-25

\section{Sagt fMRT den Therapieerfolg der KVT voraus?}

Die therapeutischen Goldstandards bei sozialer Angststörung sind kognitive Verhaltenstherapie (KVT) und Pharmakotherapie. Allerdings zeigen beide Methoden nur bei einem Teil der Patienten einen guten Therapieerfolg. Bisher existieren keine Prädiktionsinstrumente, welcher Patient respondiert oder nicht.

oziale Angststörungen (SAS), auch $S$ als soziale Phobie bezeichnet, stellen eine der häufigsten Angststörungen dar, die - bei deutlicher Ausprägung - zu einer massiven Beeinträchtigung der Betroffenen führt. Deutliche interindividuelle Response-Unterschiede auf KVT und Pharmakotherapie werden auf nicht genügend geklärte Unterschiede der genetischen, neurobiologischen und phä-

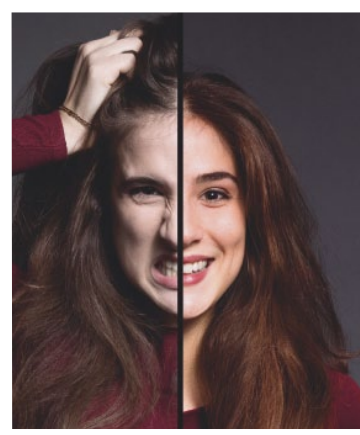

Wie sieht das Aktivierungsmuster im fMRT als Reaktion auf diese Gesichter aus?

notypischen Determinationsfaktoren der Störung zurückgeführt.

In die Studie wurden 39 Patienten mit einer SAS eingeschlossen. Vor dem Beginn einer zwölfwöchigen KVT wurde ein fMRT unter Stimulation mit angstvoll-aggressiven beziehungsweise neutralen Gesichtern durchgeführt. Das Aktivierungsmuster im fMRT vor Beginn der KVT konnte, gemeinsam mit Daten über die Schwere der Symptomatik, mehr als $40 \%$ der Varianz des späteren Behandlungserfolgs erklären, ein Ergebnis, das weit über rein klinischen Prädiktoren liegt. Das kritische Hirnareal für diese Prädiktion umfasst ein Gebiet im rechten temporo-occipitalen Assoziationskortex.

Kommentar: So interessant das geschilderte Ergebnis ist, so schwierig ist dessen Interpretation. Eine der potenziellen Erklärungsmöglichkeiten ist, dass eine verminderte
Aktivierung als Reaktion auf Gesichter mit emotionalem Inhalt im temporo-occipitalen Assoziationskortex ein Charakteristikum jener SAS-Patienten ist, die schlechter auf eine therapeutische Intervention ansprechen. Hier ergeben sich eine Reihe von weiteren Fragen: Was ist die Ursache dieser verminderten Reaktivität in diesem Gehirngebiet? Überwiegen genetische oder erworbene Ursachen? Ist die Reaktivität in diesem Kortexareal auch ein Prädiktor für den Erfolg einer pharmakologischen Intervention?

Trotz dieser ungeklärten Fragen ist es in dieser Studie zum ersten Mal gelungen, einen Prädiktor für den Behandlungserfolg zu identifizieren, vor Klärung der gerade aufgeworfenen Fragen sollte allerdings der beschriebene Befund repliziert werden.

Prof. Dr. med. Hans-Peter Volz

Doehrmann $\mathrm{O}$ et al. Predicting treatment response in social anxiety disorder from functional magnetic resonance imaging. JAMA Psychiatry 2013; 70 (1): $87-97$ 